\title{
Should oncologists be responsible for monitoring post-treatment follow-up in cancer patients?
}

\author{
Barbara Radecka ${ }^{1}$, Joanna Streb²
}

With increasing cancer rates, a steady rise is also observed in the numbers of such patients being followed-up after their treatment. This aspect of healthcare embraces cancer patients recovering from radical treatment, post-palliative treatment and frequently those patients who have exhausted the possibilities of specialist treatment. Controversies are ever ongoing about who should be responsible for monitoring post-treatment follow-up. Bearing in mind the limited numbers of specialists in clinical oncology, radiotherapy and cancer surgery, relative to GP specialists, the roles of GPs should be more boldly and clearly defined regarding the delivery of healthcare to cancer patients. Developing proficient 'healthcare models' based on collaboration between oncologists and GPs is thereby required. This article presents the arguments for justifying such a solution.

NOWOTWORY J Oncol 2016; 66, 6: 490-493

Key words: post-cancer-treatment monitoring, follow-up, recovering patients, coordinated oncology healthcare, General Practitioners

\section{Introduction}

Monitoring cancer patients recovering during posttreatment forms a significant part of delivering comprehensive healthcare in oncology. Such surveillance embraces patients after radical treatment, those having completed palliative therapy and often patients who have exhausted the means of specialist treatment. Coupled with the growing numbers of cancer patients, is a constant increase in cancer survivors. The advances made in the early detection of cancer, along with specialised therapy have led to lasting remissions or complete cures. Cancer survivors are in any case constantly monitored when their treatment is ended for over several to even dozens of years. The numbers of convalescents registered for monitoring by oncologists, as well as by GPs are constantly rising, with only a few not taking advantage of such services [1]. With the passage of time after ending treatment, visits to oncologists become reduced whereas GPs are increasingly seen more often [1, 2]. Around one third of those monitored take advantage of both these options in parallel, with the rest mostly remaining under the care of an oncologist [3]. It should however be stressed that such evidence comes from studies that are by nature observational, retrospective or they are surveys, and therefore suffer from many limitations. Random sampling studies of patients are however lacking.

The issue of post-treatment monitoring of cancer patients poses a serious challenge in the organisation and financing for all healthcare systems, no matter how they are set up and function, nor do they depend on the level of a country's economic development.

This situation has come about not merely by the increased numbers of patients covered by these services but, inter alia, by the rising costs of diagnostic testing. It should be remembered that the healthcare required by convalescing patients after radical treatment is in itself specific in nature (ie. observations aimed at establishing whether treatable relapses or other primary tumours occur and the diagnosing of late complications arising from cancer treatment) as well as for patients ending palliative treatment (interventions aimed at improving the quality of life). The

${ }^{1}$ Department of Clinical Oncology, Tadeusz Koszarowski Regional Oncology Center, Opole, Poland

${ }^{2}$ Department of Oncology, Jagiellonian University Hospital, Kraków, Poland 
problem thus arises on who should be responsible for the post-treatment monitoring of these cases.

Despite efforts over many years, no healthcare system has successfully addressed this issue. The most effective systems place onus on coordinating the services provided by the specialist and GPs as well as possibly specialist nurses. This becomes important insomuch that lifestyle recommendations and preventative measures are usually somewhat different; if coming from the oncologist then these deal with cancer, but if from the GP, then other $21^{\text {st }}$ century diseases are dealt with [4]. International Scientific Societies (ie. ESMO, ASCO and ACS) have attempted to agree a consensus for post-treatment monitoring of cancer patients, taking into account the disease characteristics and its treatment course although this can be difficult in some instances, because the appropriate scientific data can be lacking. It is important that such recommendations consider the type of healthcare system operating in a given country so that they can become incorporated into everyday practice [5].

Poland lacks a system of coordinated healthcare for cancer survivors. Such care is provided by oncologists or other specialists involved in cancer treatment (e.g. radiotherapists, laryngologists, urologists, haematologists etc.). Thus, there exists an urgent need for creating a system based on the collaboration between specialists and GPs, with both sides mutually complementing each other.

\section{Arguments for making the oncologist responsible for monitoring}

It is universally recognised that after intensive and usually long-lasting treatment for cancer, the patient undergoes monitoring by an oncologist where, apart from interviews and physical examination, additional testing is performed (laboratory and imaging tests). During treatment patients can often lose contact with their GPs, where their doctor now becomes the oncologist and all health issues are now dealt as part of specialised healthcare [6]. This creates a certain mental barrier for returning back to the healthcare provided by the GP when treatment ends, as the patient is still expecting healthcare to be delivered by the specialist $[7,8]$. The patient expects that tests performed during follow-up will also lessen their anxiety about any possible relapse. Some studies indicate that patients have more confidence in specialists, and when specialists and GPs are compared, then patients tend to favour the former in respect of being more satisfied [9]. Nevertheless, there is plenty of evidence showing there are no significant differences in detection rates of relapses, quality of life during follow-up, depression and anxiety between oncologists/cancer surgeons when compared to GPs $[4,10]$.

It should be noted that GPs does not often see a cancer patient. According to the National Cancer Registry from 2013 , there are around 900,000 people in Poland diagnosed with cancer, with almost half of these having been diagnosed within the last 5 years [11]. Under the conditions in Poland, it is also noteworthy that on average a GP has around 2,000-2,500 patients under their care, where of these, statistically speaking, one can expect 50-60 persons who had been diagnosed with cancer. Taking into account the morbidity (ie. around 160,000 new cases annually), the GP has a chance for diagnosing 8-10 cancers and to confirm 4-5 deaths arising from cancer per year [11]. Such outcomes do not help GPs in acquiring experience for delivering healthcare to cancer patients.

This problem is already apparent at the undergraduate level, where the role of the oncologist is marginalised and the study time devoted to this subject is low together with the commonly met attitude that oncology can anyway be taught in almost any clinic or any other clinical departments. At the post-graduate level things are little better, where specialisations in internal medicine or family medicine do not include an oncology placement. A GP therefore does not get the opportunity for gaining the relevant knowledge about oncology, and yet cancer is the second highest cause of mortality in Poland. This results in low detection rates of early stage cancer and omissions made in diagnoses.

Another doubtful issue is whether the knowledge of complications after cancer treatment is adequate. Studies indicate that GPs can very rarely diagnose complications after chemotherapy and likewise polyneuropathy, pulmonary fibrosis, heart failure, early menopause or relapsed tumours [12]. Oncologists do not provide patients with information in their discharge notes for GPs concerning the risk of complications; patients are in fact not practically observed with such events in mind. In cancer survivors, invariably of young age, there are also elevated risks of disorders arising in the metabolism of adipose tissue (increased production of pro-inflammatory adipokins) and muscle (increased insulin resistance) and endocrine disorders (pituitary-hypothalamic axis damage and deficiencies in growth hormone, oestrogens, testosterone or thyroid hormones [13]. At the same time, GPs and some specialists in other areas, have insufficient understanding of patient needs during their convalescence. Many myths are at fault here, such as adverse reactions to changes in the weather to rehabilitation. Although, as a consequence, cancer treatment reduces physical activity and fitness and that physical rehabilitation (at health spa conditions) improves fitness and lessens feeling of fatigue, doctors reluctantly recommend rehabilitation and even consider this to be counter-productive.

The purpose of follow-up after cancer treatment is also to analyse long-term outcomes in treatment through survival times and general health status. Long-term follow-ups of patients supply a broad knowledge-base on this topic. This particularly applies to persons who had suffered illness 
at childhood and, on becoming cured, had been followedup for 20, 30 or more years [14].

All this goes to show that follow-ups cannot be conducted without an oncologist being present. It should be emphasised that testing schemes should be individually tailored, especially in patients undergoing palliative treatment in whom further curative treatment is possible.

\section{Arguments for making the GP responsible for monitoring}

With the steady increase in cancer morbidity and the growing numbers of post-treatment cancer patients, the question arises as to who should be responsible for monitoring the follow-up. Given the small numbers of specialists in clinical oncology, radiotherapy and cancer surgery in Poland, when compared to GP specialists, the defined roles of GPs should be put forward more boldly in delivering healthcare to cancer patients. Notwithstanding the conviction that post-treatment patients are better off being monitored by an oncologist, it should be clearly stated that there are insufficient numbers of both specialist oncology centres and oncologists in Poland; at present there are 700 clinical oncologists and 29,000 GPs. The aforementioned data from the National Cancer Registry, along with the observed everyday practice of oncologists indicate that specialist out-patient healthcare in oncology centres is and will be increasingly burdened by the requirements for undertaking follow-ups.

It is noteworthy that patient follow-up after radical cancer treatment is, above all else, intended to discover if there have been any relapses or that the state of any subsequent primary tumour found allows treatment to re-commence; such procedures can be undertaken by GPs. Post-treatment patients commonly expect to undergo regular imaging tests to 'assure themselves that there is no disease progression'. This applies to both those who had undergone radical treatment as well as those after palliative therapy. An important aspect of follow-up is to maintain an appropriate balance between patient expectations and a realistic assessment of the value that any given diagnostic tests may bring; as well as the costs involved. It is also important to recognise that there is a lack of clear, unequivocal and accepted standards in follow-up which make doctors, whether in oncology centres or GPs, vulnerable to being accused of failing to provide adequate care [5].

Many cancer survivors have experienced various comorbidities and as a result they regularly see their GPs because waiting times to specialist clinics are much longer. It is the GP who has full knowledge on the patient's health status and is responsible for the entire treatment of co-morbidities; knowing 'his/her' patients well. Delivering such healthcare may equally consist of monitoring during post-cancer treatment follow-up. It is the GP who is more often likely to see the patient than the specialist to conduct interviews or physical examinations.

Even though specialist teaching in family medicine does not include placement at an oncology department, nonetheless oncology constitutes part of the placement for palliative care and in hospices, where future GPs will have had experience of cancer patients. Within the remit of specialist teaching for family medicine, doctors also take part in lectures and seminars on oncology. Indeed, basic training in family medicine takes 4 years for a doctor not yet specialised, where they will on numerous occasions come into contact with cancer patients. Physicians working as GPs can take advantage of the annex to their contract with the NFZ (Polish National Health Fund) regarding listings of permissible laboratory and imaging tests, but this list insufficiently covers follow-up monitoring post cancer treatment. In accordance with this contract, GPs are not allowed to issue referrals for computer tomography (CT) scans, mammographies or breast ultrasound. This severely limits any follow-ups after adjuvant therapy for breast or colonic cancer and thereby obliges the patient to see a specialist. A simple legal amendment could clearly alter this, since the aforementioned cancers (i.e. breast and colon) belong to the most commonly occurring cancers in Poland and that most cases are treated radically which requires many years of follow-up.

When making diagnoses and in treating complications after cancer treatment, both GPs and oncologists seek consultations with other specialists that may include neurologists, cardiologist, endocrinologists or orthopaedicians and in any case the healthcare delivered by the GP or oncologist should be comparable. Studies indicate that GPs are capable of carrying out follow-ups after cancer treatment in a timely and patient-safe fashion $[15,16]$.

\section{Conclusions}

Appropriate management of cancer survivors followup poses a serious challenge in terms of organisation and financial resources to the healthcare system, above all else because the number of such patients is constantly rising and where they feel completely lost in this system. Studies have indicated that one third of cancer survivors don't know, who actually bears responsibility for their healthcare [9]. The experiences of many countries show that the most effective solutions are those that emphasise coordinated healthcare between the specialist and GP (including the family doctor) together with perhaps specialist nurses. Poland needs to urgently create a system of coordinated healthcare in this field embracing equally the oncologist as well as the GP.

This article presents a list of arguments that justify such a solution. The Polish legislation popularly known as 'The Oncology Package' assumes that patients treading'the path of rapid diagnostics and cancer treatment' will after its con- 
clusion/termination return to being under the healthcare of their GP [17]. The proposed solution in the legislation is unfortunately not supported by any changes in the financing of post cancer treatment follow-up. It is difficult to expect that this care will be taken up by GPs. In practice, the patient however remains under the care of the oncologist, where cancer centres are still wrestling with the so-called problem of 'overspending' regarding specialist out-patient healthcare of which patient visits and follow-up testing forms a significant part. Creating a system of coordinated healthcare also requires introducing changes in the underand post-graduate education of doctors. One of the mainstays of such a system could become the nurse. Engaging this group of professionals has proved successful in diabetic care, where nurses have made a great contribution, inter alia, in educating patients. It is worth taking advantage of these solutions, particularly since the law has widened the range of nurse competencies, for instance in the issuing of prescriptions for medicines.

Within recent years, dynamic developments in telemedicine have been witnessed. In some specialisations, this has enabled effective monitoring, at a distance, of the patient's health status which is particularly important at a time when the population is aging. Various types of tele-monitoring are currently under investigation that use electronic devices and even social media, which include applications for cancer patients. This phenomenon is worth observing with a view for introducing such solutions in Poland.

\section{Conflict of interest: none declared}

\section{Barbara Radecka, MD, PhD}

Opole Oncology Centre

ul. Katowicka 66 a

45-061 Opole, Poland

e-mail:brad@onkologia.opole.pl

Received \& Accepted: 15 Aug 2016
Based on the presentation at the IV Annual Conference of the Nowotwory Journal of Oncology, 'Oncological Debates', held in Warszawa, 8-9th April 2016

\section{References}

1. Snyder CF, Earle CC, Herbert RJ. Trends in follow-up and preventive care for colorectal cancer survivors. J Gen Intern Med 2008; 23: 254-259.

2. Del Giudice L, Bondy SJ, Maarten S et al. Physician care of cancer patients: ICES atlas. Ontario, 2006; 162-174.

3. Hewitt M, Bamundo A, Day Y. Perspectives on post-treatment cancer care: qualitative research with survivors, nurses, and physicians. J Clin Oncol 2007; 25: 2270-2273.

4. Grunfeld E, Earle CC. The interface between primary and oncology specialty care: treatment through survivorship. J Natl Cancer Inst Monographs 2010; 2010: 25-30.

5. Jassem J, Duchnowska R, Kawecki A et al. Badania kontrolne po leczeniu w najczęstszych nowotworach litych u dorosłych. Nowotwory J Oncol 2014; 64: 415-435.

6. Rosenblatt RA, Hart LG, Baldwin LM et al. The generalist role of specialty physicians: is there a hidden system of primary care. JAMA 1998; 279 : 1364-1370.

7. Dworkind M, Shvartzman P, Adler PS et al. Urban family physicians and the care of cancer patients. Can Fam Physician 1994; 40: 47-50.

8. Mc Whinney IR, Hoddinott SN, Bass MJ et al. Role of the family physician in the care of cancer patients. Can Fam Physician 1990; 36: 2183-2186.

9. Miedema B, MacDonald I, Tatemichi S. Cancer follow-up care. Patients perspectives. Can Fam Physician 2003; 49: 890-895.

10. Wattchow DA, Weller DP, Esterman A et al. General practice vs surgical-based follow-up for patients with colon cancer: randomised controlled trial. Br J Cancer 2006; 94: 1116-1121.

11. Krajowy Rejestr Nowotworów, 2013.

12. Nekhlyudov L, Aziz NM, Lerro $C$ et al. Oncologists' and primary care physicians' awareness of late and long-term effects of chemotherapy: implications for care of the growing population of survivors. J Oncol Pract 2014; 10: e29-e36.

13. Reilley MJ, Jacobs LA, Vaughn DJ et al. Health behaviors among testicular cancer survivors. J Community Support Oncol 2014; 12: 121-28.

14. Armstrong GT, Liu Q, Yasui Y et al. Late mortality among 5-year survivors of childhood cancer: a summary from the Childhood Cancer Survivor Study. J Clin Oncol 2009; 27: 2328-2338.

15. Grunfeld E, Fitzpatrick R, Mant D et al. Comparison of breast cancer patients satisfaction with follow up in primary care versus speciatist care: results from a randomized controlled trial. Br J Gen Pract 1999; 49: 705-710.

16. Grunfeld E, Mant D, Vessey MP et al. Specialist and general practice views on routine follow-up of breast cancer patients in general practice. Fam Pract 1995; 12: 60-65.

17. Ustawa z dnia 27 sierpnia 2004 r. o świadczeniach opieki zdrowotnej finansowanych ze środków publicznych (Dz.U. 2014 poz. 1138, art. 32b, ust. 3). 